\section{BRAZIULIAN JOURNAL}

OF MEDICAL AND BIOLOGICAL RESFARCH

www.bjournal.com.br
ISSN 0100-879X

Volume 44 (7) 606-728 July 2011

BIOMEDICAL SCIENCES

AND

CLINICAL INVESTIGATION

Braz J Med Biol Res, July 2011, Volume 44(7) 606-617

doi: 10.1590/S0100-879X2011007500068

Molecular and cellular pathogenesis of autosomal dominant polycystic kidney disease

A.P. Bastos and L.F. Onuchic

The Brazilian Journal of Medical and Biological Research is partially financed by
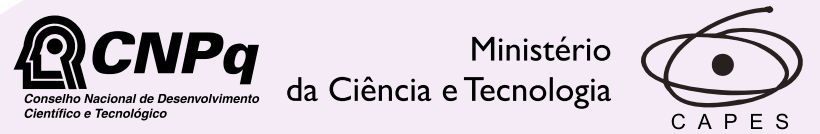

Ministério da Educação
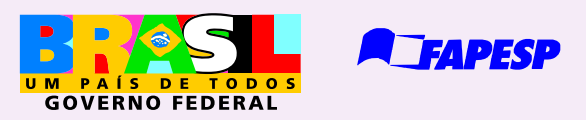

Institutional S ponsors
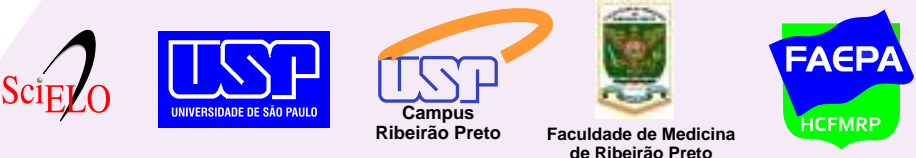


\title{
Molecular and cellular pathogenesis of autosomal dominant polycystic kidney disease
}

\author{
A.P. Bastos and L.F. Onuchic \\ Disciplina de Nefrologia, Faculdade de Medicina, Universidade de São Paulo, São Paulo, SP, Brasil
}

\begin{abstract}
Autosomal dominant polycystic kidney disease (ADPKD) is one of the most common human life-threatening monogenic disorders. The disease is characterized by bilateral, progressive renal cystogenesis and cyst and kidney enlargement, often leading to end-stage renal disease, and may include extrarenal manifestations. ADPKD is caused by mutation in one of two genes, $P K D 1$ and PKD2, which encode polycystin-1 (PC1) and polycystin-2 (PC2), respectively. PC2 is a non-selective cation channel permeable to $\mathrm{Ca}^{2+}$, while PC1 is thought to function as a membrane receptor. The cyst cell phenotype includes increased proliferation and apoptosis, dedifferentiation, defective planar polarity, and a secretory pattern associated with extracellular matrix remodeling. The two-hit model for cyst formation has been recently extended by the demonstration that early gene inactivation leads to rapid and diffuse development of renal cysts, while inactivation in adult life is followed by focal and late cyst formation. Renal ischemia/reperfusion, however, can function as a third hit, triggering rapid cyst development in kidneys with Pkd1 inactivation induced in adult life. The PC1-PC2 complex behaves as a sensor in the primary cilium, mediating signal transduction via $\mathrm{Ca}^{2+}$ signaling. The intracellular $\mathrm{Ca}^{2+}$ homeostasis is impaired in ADPKD, being apparently responsible for the cAMP accumulation and abnormal cell proliferative response to cAMP. Activated mammalian target for rapamycin (mTOR) and cell cycle dysregulation are also significant features of PKD. Based on the identification of pathways altered in PKD, a large number of preclinical studies have been performed and are underway, providing a basis for clinical trials in ADPKD and helping the design of future trials.
\end{abstract}

Key words: Autosomal dominant polycystic kidney disease; PKD1 gene; PKD2 gene; Polycystins 1 and 2; Cystogenesis; Primary cilium

\section{Introduction}

Autosomal dominant polycystic kidney disease (ADPKD) is the most common monogenic kidney disease, affecting all races. Though most patients seek medical attention due to its renal manifestations, ADPKD is a systemic disorder, including a variety of extrarenal abnormalities, which may be translated into cystic or non-cystic phenotypes (1). While the cystic manifestations are characterized by age-dependent occurrence of bilateral, multiple renal cysts and cysts that may be present in the liver, seminal vesicles, pancreas, arachnoid membrane, and spinal meninges, the noncystic alterations include vascular anomalies mainly represented by intracranial aneurysms and dolichoectasias, aortic root dilatation and mitral valve prolapse, as well as abdominal wall hernias and colon diverticula (2).

\section{Epidemiology and natural history}

ADPKD affects 1:400 to 1:1000 people and is associated with virtually complete penetrance. Its clinical manifestations usually appear in the 3rd to 4th decade of life, although in $1-2 \%$ of the cases they are seen in infancy or early childhood. Only half of the patients reach the age of 58 years without end-stage renal disease (ESRD). The disease, in fact, accounts for $4.4 \%$ or more of patients in renal replacement therapy worldwide, being the third main cause of ESRD if each type of glomerulopathy is considered individually.

The severity of the disease, the age of onset of ESRD, and the extrarenal manifestations vary widely among affected individuals. Factors related to a higher rate of renal disease progression, in turn, include PKD1 (polycystic kidney disease 1) as the mutated locus, increased kidney size and growth rate (3), systemic hypertension, early onset of hematuria, proteinuria, and possibly male gender for ADPKD1. In addition to the loss of renal function, patients may also develop other kidney-related manifestations, mainly represented by hypertension, chronic abdominal

Correspondence: L.F. Onuchic, Departamento de Clínica Médica, Faculdade de Medicina, Universidade de São Paulo, Avenida Dr. Arnaldo, 455, Sala 4304, 01246-903 São Paulo, SP, Brasil. Fax: +55-11-3061-8361. E-mail: lonuchic@usp.br

Received February 27, 2011. Accepted April 18, 2011. Available online May 27, 2011. Published July 25, 2011. 
or flank pain, nephrolithiasis, hematuria, concentration dysfunction, cyst infection, non-cystic urinary tract infection, and cyst hemorrhage.

\section{Pathology}

Cyst growth is a gradual process that can lead to the formation of enormous cystic kidneys in ADPKD, as well as to a markedly enlarged liver (Figure 1). Fluid-filled cysts of sizes that can vary from millimeters to more than $10 \mathrm{~cm}$ are distributed throughout the renal parenchyma, though non-cystic areas can be observed in young patients. The cyst fluid may be clear or cloudy, watery or thick. Previous cystic bleeding can turn the fluid dark due to blood degradation, and often cyst walls can evolve with calcifications.

Cyst formation is a focal process in ADPKD, and reports based on human kidney microdissection suggest that they are derived from only 1 to $2 \%$ of the nephrons. Interestingly, after reaching a $\sim 2 \mathrm{~mm}$ in diameter, the cyst tends to disconnect from the originating tubule. While cysts may arise from all segments in ADPKD, apparently those derived from collecting ducts appear in higher numbers and are larger than the ones of other origins. Most of the cysts are lined by a single layer of poorly differentiated epithelial cells, though a minority of them can be lined by a significantly hyperplastic epithelium. Interstitial inflammation, in turn, is usually observed and can lead to cyst-surrounding fibrosis in advanced ADPKD kidneys.

\section{Genetics}

ADPKD is a genetically heterogeneous disorder caused by mutations in one of two genes: PKD1 (located on 16p13.3) and PKD2 (polycystic kidney disease 2, mapped to $4 q 21$ ). Although some unlinked families have been reported, further analysis of one of them has revealed bilineal inheritance of PKD1 and PKD2 heterozygous mutations, making uncertain the existence of a third ADPKD locus (4). Mutations in the PKD1 gene are responsible for $\sim 85 \%$ of the disease cases, whereas mutations in PKD2 account for $\sim 15 \%$ of them. It should be noted that, while the renal and extrarenal manifestations are similar in both genetic forms, when caused by mutations in PKD1 the disease is associated with a more severe clinical course than when caused by mutations in PKD2 (5). This difference seems to be dependent on the development of a larger number of cysts early in life in ADPKD1, and not on a higher cyst growth rate (6).

\section{The PKD1 and PKD2 genes}

PKD1 includes 46 exons distributed throughout a $~ 52-$ $\mathrm{kb}$ genomic segment. The gene gives rise to a $14.2-\mathrm{kb}$ mRNA, with an open reading frame of $\sim 12.9 \mathrm{~kb}(7)$. An interesting feature of $P K D 1$ is the fact that its 5 ' portion is positioned in a region duplicated several times on chromosome 16, making its distinction from highly homologous pseudogenes a relatively complex process at the patient level. PKD2, on the other hand, spans a $\sim 68-\mathrm{kb}$ genomic region and comprises 15 exons. This gene encodes a 5.4-kb transcript that encompasses a $2.9-\mathrm{kb}$ open reading frame (8).

Hundreds of different mutations in both genes can cause ADPKD. While most of the pathogenic mutations lead to truncation of the respective gene product, a considerable number of mutations are missense.

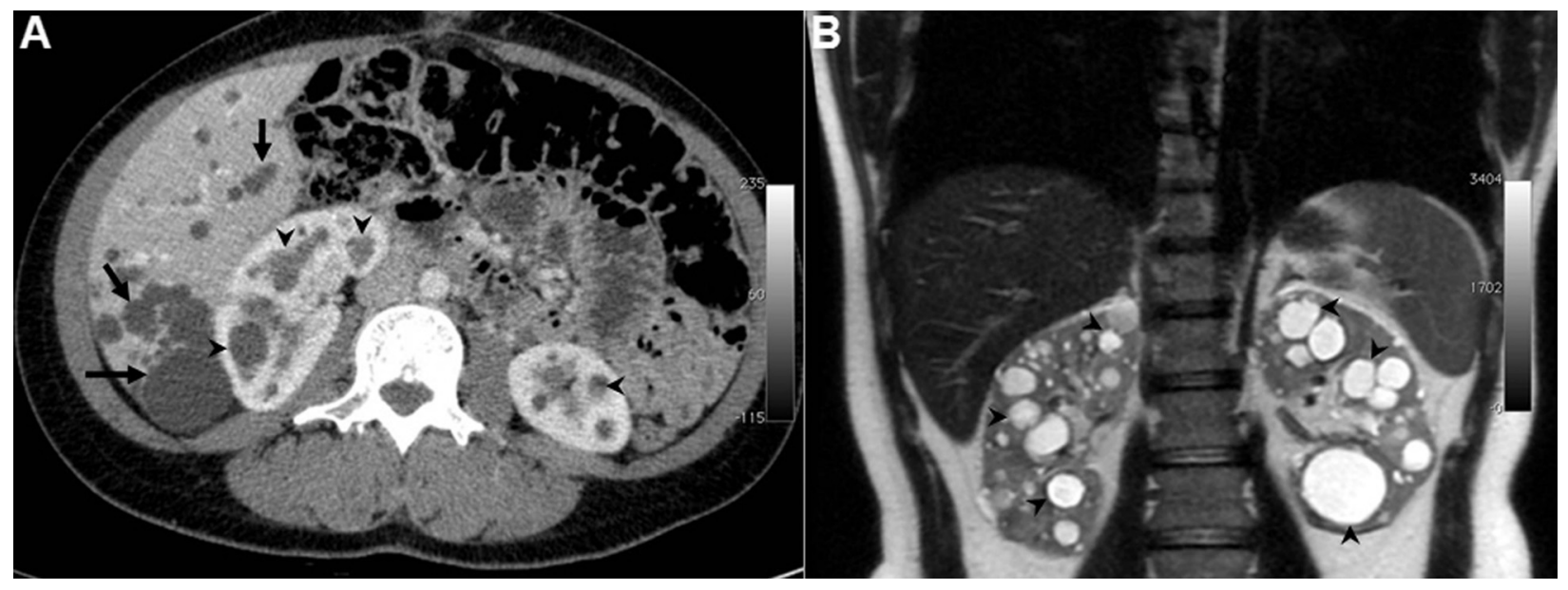

Figure 1. Autosomal dominant polycystic kidney disease (ADPKD). A, A 39-year-old ADPKD female patient with enlarged, polycystic kidneys and liver; computed tomography with iodinated contrast, showing multiple hepatic (arrows) and renal cysts (arrowheads) of variable sizes. B, A 32-year-old male patient with ADPKD; magnetic resonance with gadolinium contrast, displaying bilateral multiple cysts (arrowheads) distributed throughout the kidneys. 


\section{Phenotype variability and genotype-phenotype correlations}

ADPKD shows significant clinical variability. This is observed not only among families but also among members of the same family, in whom the germline mutation is the same. The cyst formation in the kidneys, moreover, consists of a focal process. In addition to effects associated with the locus involved in the disease (5), the influence of modifier loci and the involvement of environmental factors seem to also explain the observed variation. Mosaicism is described as another cause of phenotypic variability within a family, being a potential modulator of disease severity. Comparative analysis between siblings and monozygotic twins supports indeed the influence of modifier genes on the progression of renal disease. Furthermore, the increased risk of recurrence of early-onset ADPKD in affected families is suggestive of a common family background for modifier genes for early and severe phenotype. Variants in other genes associated with renal cystic diseases might contribute to this process. Contiguous deletion of the adjacent genes PKD1 and TSC2 (one of the loci mutated in tuberous sclerosis), in fact, leads to early-onset PKD associated with clinical manifestations of tuberous sclerosis. In the search for other potential ADPKD modifier genes, the angiotensin-I-converting enzyme I/D polymorphism was analyzed but most investigators did not find association with early progression to ESRD. It is expected, however, that analyses of quantitative trait loci may make a significant contribution to this process. Finally, based on the molecular mechanism that underlies cystogenesis in ADPKD that will be discussed in this review, the frequency and the time in life when somatic mutations occur in PKD1/PKD2 appear to also be critical for the course of renal disease.

The nature of the germline mutation does not markedly correlate with the disease phenotype in either ADPKD1 or ADPKD2. Also, no clear correlations were observed for the location of $P K D 2$ mutations. The position of PKD1 mutations, however, was shown to be relevant: patients with mutations in the 5' region of the gene were associated with an earlier onset of ESRD and a higher prevalence of intracranial aneurysms than patients with mutations positioned at the 3' portion of $P K D 1$. A recent study, moreover, has reported the existence of hypomorphic or incompletely penetrant PKD1 alleles (9). While one of these alleles may determine the development of mildly cystic kidneys, the combination of two of them can lead to classical to severe phenotypes and association with an inactivating allele may result in early-onset ADPKD.

\section{Polycystins 1 and 2, the PKD1 and PKD2 gene products}

The PKD1 gene encodes polycystin-1 (PC1), a large integral membrane glycoprotein with 4303 amino acids (aa) and an estimated molecular mass of $\sim 460 \mathrm{kDa}$ (Figure 2). This protein contains $a>3000$-aa extracellular portion with a complex combination of domains (Figure 2) that support protein-protein and protein-carbohydrate interactions. Sixteen immunoglobin-like domains named PKD repeats are part of this set, being followed by a receptor for egg-jelly (REJ) domain and a G-protein-coupled receptor proteolytic site (GPS). While the PKD repeats are apparently important for molecular interactions and the REJ domain appears to play significant regulatory roles, the GPS domain is likely to be involved in the process of PC1 activation. This cleavage occurs immediately before the first transmembrane (TM) domain, resulting in $\mathrm{N}$ - and $\mathrm{C}$-terminal fragments. $\mathrm{PC} 1$ also includes 11 TM domains and a short 197-aa intracellular C-terminus. This intracytoplasmic tail contains a coiledcoil domain and a G-protein-binding domain, playing an essential role in signal transduction, as well as an aminoacid sequence that may contribute to its ubiquitin-mediated degradation. Altogether, the PC1 structural features predict the protein to function as a receptor and that it may also act as an adhesion molecule, mediating cell-cell and cellextracellular matrix interactions.

Polycystin-2 (PC2), the PKD2 gene product, is also an integral membrane glycoprotein (Figure 2). This $\sim 110-k D a$ protein is formed by 968 aa and is predicted to contain six TM domains and intracytoplasmic $\mathrm{N}$ - and $\mathrm{C}$-termini (Figure 1). Interestingly, $P C 2$ shows homology to the last six TM domains of PC1, and PC1 and PC2 comprise a subfamily of transient receptor potential (TRP) channels. The PC2 $\mathrm{N}$-terminus includes a dimerization domain and a ciliumtargeting motif (10). Its C-terminal tail, in turn, contains an EF-hand calcium-binding motif and a coiled-coil domain responsible for protein-protein interactions. PC2, also referred to as TRPP2, and PC1 (TRPP1) physically interact by their C-termini, a binding process involving the PC1 coiled-coil domain as well as residues present in a PC2 coiled-coil. PC2 is a non-selective cation channel permeable to $\mathrm{Ca}^{2+}$ whose activity is regulated by $\mathrm{PC} 1$, a process that constitutes the polycystin-signaling complex. This physical interaction is thought, therefore, to play a key role in intracellular $\mathrm{Ca}^{2+}$ homeostasis. In addition, both polycystins have been shown to interact with a number of different proteins. It must be noted, then, that the outlined features are consistent with multifunctional roles for PC1 and PC2.

PC2 is mainly expressed in the endoplasmic reticulum (ER), but different studies have shown that it is also found in the plasma membrane, primary apical cilium, centrosome, and mitotic spindles. PC1, in turn, shares the localization with PC2 in the primary cilium, plasma membrane and potentially in the ER. In mature renal tubules, PC1 is expressed in the basolateral membrane at sites of cell-cell interactions, in junctional structures identified as desmosomes and adherens junctions. A C-terminal fragment has also been detected in the cell nucleus (11). PC1 has been found, moreover, in cytoplasmic vesicles. 
Interestingly, PC1 and PC2 have been abundantly found in a fraction of urine exosomes. While PC2 shows significant expression during development and in the mature kidney, PC1 expression is highest during kidney development but low in the adult kidney.

The PC2 subcellular transport and localization are regulated by phosphorylation processes and interaction with adapter proteins. Phosphorylation by casein kinase 2 can lead it to bind to phosphofurin acidic cluster sorting protein-2 (PACS-2), a connector protein bound to COPI, or to phosphofurin acidic cluster sorting protein-1 (PACS-1), a connector molecule bound to activator protein 1 (AP-1) (12). While the physical interaction with PACS-2 mediates its trafficking to the ER, its binding to PACS-1 takes it to the Golgi/trans-Golgi network. PC2 dephosphorylation by protein phosphatase $2 \mathrm{~A}$, on the other hand, can release it from these interactions and translocate PC2 to the plasma membrane. It must be mentioned that translocation of PC2 to the plasma membrane is also mediated by its binding to PC1 (13). A recent study, in turn, suggests that the localization of PC1 at the plasma membrane is stimulated by PC2 and by cleavage at the GPS (14).

\section{PC1 cleavage: structural basis and biological implications}

The PC1 cleavage capacity and the generation of an $\mathrm{N}$-terminal fragment reflect relevant and specific functional properties. While the $\mathrm{N}$ - and $\mathrm{C}$-terminal fragments remain tethered after cleavage at the GPS domain, they may detach depending on appropriate stimulation. In recent years, a series of observations has defined the functional particularities and biological implications related to appropriate GPS-mediated PC1 cleavage. While mice homozygous for $P k d 1$ null mutations die prenatally with massively cystic kidneys, pancreatic cysts, skeletal defects (15), and cardiac

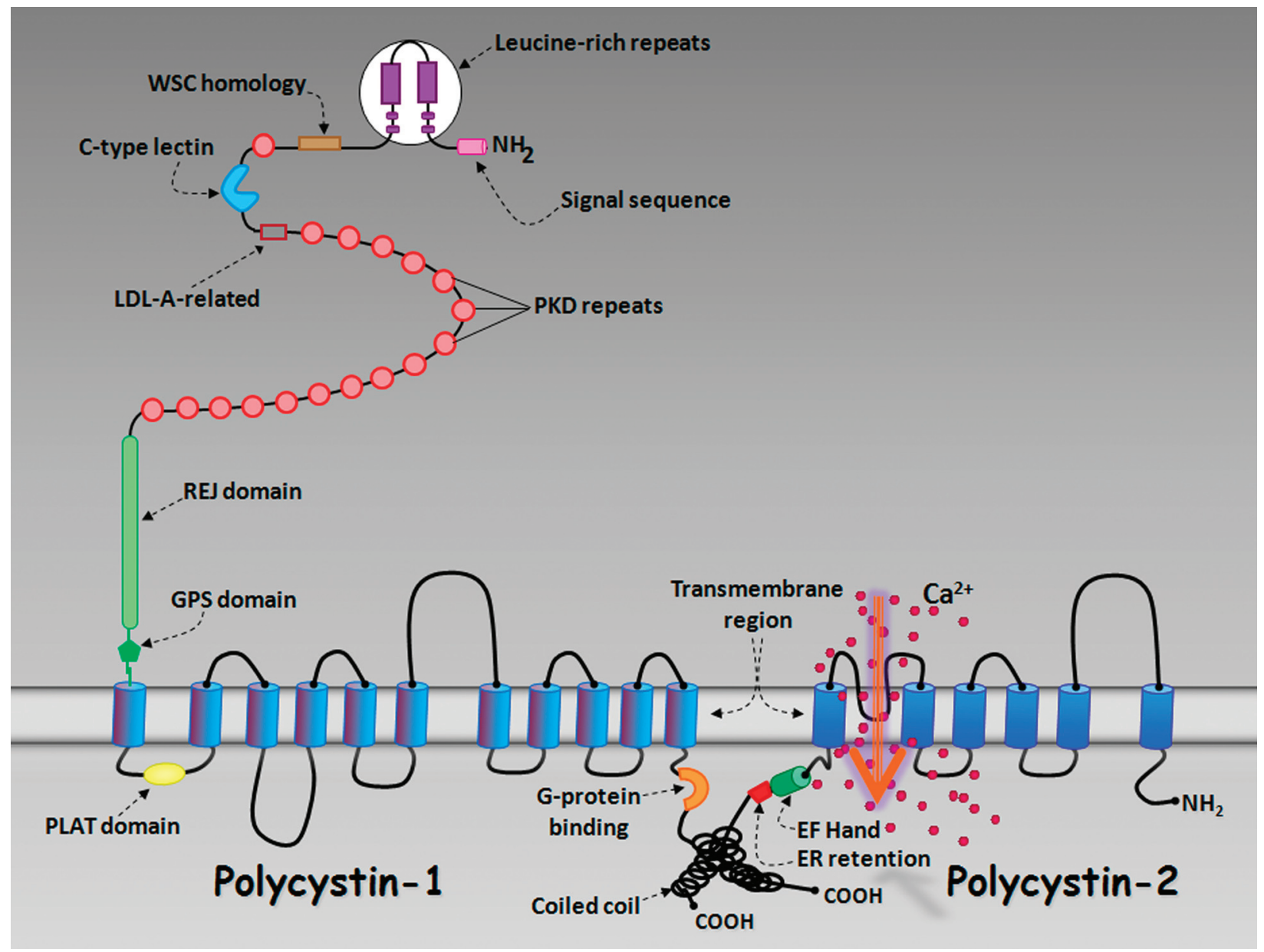

Figure 2. Domain structure of polycystin-1 and polycystin-2. The protein domains are identified in the figure. ER = endoplasmic reticulum; GPS = G-protein-coupled receptor proteolytic site; LDL-A = low-density lipoprotein A; PLAT = polycystin-1, lipooxygenase, alpha toxin; REJ = receptor for egg-jelly; WSC = cell-wall and stress-response component. 
and vascular abnormalities, homozygotes for a mutation that prevents PC1 cleavage but keeps the expression of its non-cleavable form develop a postnatal distal nephron cystic phenotype (16). This renal cystic phenotype, in turn, is rapidly progressive, leading to significantly enlarged kidneys, loss of renal function, and death of the vast majority of animals by 1 month of age. These findings suggest that the non-cleavable form of PC1 is critical for embryogenesis, whereas the protein cleavage is essential for postnatal development and maintenance of distal nephron segments.

Different studies support the migration of a PC1 Cterminal fragment to the cell nucleus. According to one of the models, a mechanical stimulus upon primary cilia promotes cleavage of the protein intracytoplasmic terminus, releasing a fragment with a nuclear localization motif (17). Once in the nucleus this fragment binds $\beta$-catenin, leading to the inhibition of the Wnt signaling canonical pathway. The other model suggests that, under regular luminal fluid flow, PC1 retains the non-activated signal transducer and activator of transcription (STAT6) in the cilia (11). In the absence of tubular fluid flow, however, the PC1 C-tail is cleaved, releasing a fragment that includes its final portion. This fragment then interacts with STAT6 and a co-activator, promoting an increase in transcription in the nucleus.

\section{Pathogenesis of autosomal dominant polycystic kidney disease}

\section{Mechanisms involved in cyst formation}

Analysis of DNA samples extracted from individual cyst-lining epithelia of affected kidneys provided essential elements for the processes of elucidating the molecular basis of cystogenesis and explaining disease variability (18). These studies revealed that ADPKD cysts are monoclonal and suggested that, although the disease inheritance pattern is dominant, the mechanism of cyst formation in ADPKD is recessive at the cellular/molecular level. According to this two-hit model, the germline mutation constitutes the first hit, while the second event is represented by a somatic mutation in the previously normal allele. Subsequent studies have shown that this model applies to both ADPKD1 and ADPKD2, as well as to renal and liver cysts.

Genetically modified orthologous mouse models of ADPKD supported the proposed model. Null homozygosity for $P k d 1$ or $P k d 2$ - the mouse orthologues of PKD1 and $P K D 2$ - resulted, indeed, in severe cystic formation and embryonic lethality $(15,19)$. Pkd1 or $P k d 2$ heterozygotes, on the contrary, developed only a few cysts later in life, mainly in the liver. It must be pointed out, however, that cyst formation may occur even in the absence of complete inactivation of Pkd1, since mice with two Pkd1 hypomorphic alleles developed renal cysts in the presence of decreased levels of PC1 (20). These mice with reduced PC1 expression, however, were perinatally viable. Interestingly, compound heterozygous $P k d 2^{\mathrm{WS} 25 /-}$ mice, carrying a $P k d 2$ allele that is prone to genomic rearrangement and consequent generation of a null allele, are born live but develop severely polycystic kidneys during adulthood (19). Finally, a chimeric mouse model generated by aggregation of $P k d 1 \%$ embryonic stem cells to $P k d 1^{+/+}$LacZ ROSA26 morulae survived beyond one month (21). In this case, although the cysts were initially mosaics containing $P k d 1^{-/-}$and $P k d 1^{+/+}$cells, over time the cell content gradually became predominantly $\mathrm{Pkd1}^{-/-}$. It should be mentioned, however, that alternative $P k d 1$ and $P k d 2$ dosage mechanisms also appear to lead to cystogenesis. Indeed, a Pkd1 transgenic mouse model, characterized by $P k d 1$ overexpression, developed renal cysts (22). A generated Pkd2-overexpressing transgenic mouse also developed renal cysts, as well as increased cell proliferation, apoptosis and v-raf murine sarcoma viral oncogene homolog B1 (B-Raf)-dependent extracellular signal-regulated protein kinase (ERK) activation (23).

The mechanism of cyst formation in ADPKD, however, has been comprehensively analyzed in recent years, improving the originally proposed two-hit model. Induced conditional knockout models have allowed, indeed, to evaluate in detail the effect of time on Pkd1 inactivation. According to a key study, when $P k d 1$ inactivation occurred before $\mathrm{P} 13$ the mice developed a rapidly progressive renal cystic phenotype, whereas later inactivation was followed by very late cyst formation (24). These findings suggest that the biological consequences of $P k d 1$ inactivation are determined by a developmental switch that signals the end of the kidney maturation process. Along this line, the cystforming effect of kidney-specific inactivation of Kif3a, a gene that encodes an intraflagellar transport motor protein, was also dependent on the time when inactivation was induced (25). When occurring at birth, Kif3a inactivation led to the loss of the apical primary cilium and a rapid renal cyst formation, while when induced in adulthood the inactivation determined loss of primary cilia but not a rapid development of cysts. Another study reported that the induction of $P k d 1$ inactivation in newborn mice led to fast and massive renal cyst formation, while the induction of gene inactivation in adult animals was followed by a considerably mild cystic phenotype (20). These investigators suggested that Pkd1 deficiency does not initiate sufficient autonomous cell proliferation for significant cyst formation. In a context of low cell proliferation, therefore, as observed in the adult kidney, the inactivation of $P k d 1$ would not meet the cell environment required for rapid and diffuse cystogenesis.

In a more recent study, Takakura et al. (26) demonstrated in an induced knockout model that inactivation of $P k d 1$ in the 5 th week of life resulted in the formation of focal cysts 6 to 9 weeks after knockout induction, while Pkd1 inactivation induced in the 1st week of life was accompanied by a massive development of cysts 6 weeks after gene inactivation. These findings corroborate previous observations, supporting the hypothesis that inactivation of $P k d 1$ would not be sufficient to initiate the necessary cell proliferation 
for significant cyst formation in the adult kidney. These data led the investigators to propose that the rapid development of cysts in the mature kidney might require a third hit in addition to the inactivation of both Pkd1 alleles. The nature of this extra blow, in turn, could be genetic or nongenetic. A subsequent study from the same group was able to prove this point, showing that renal ischemia/reperfusion (IR) can behave as a third hit for cyst formation in adult kidneys (27). Mice submitted to $P k d 1$ inactivation at the age of 5 weeks and to unilateral renal IR at 8 weeks had their kidneys analyzed at $12-14$ weeks. This analysis revealed that the post-ischemia kidneys were significantly cystic, a phenotype that was not observed in the kidneys not submitted to IR. Working with another Pkd1-deficiency model, Bastos et al. (28) have also shown an additional-hit effect of IR. In this case, Pkd1-haploinsufficient mice submitted to renal IR developed microcysts in their kidneys 6 weeks after the insult, alterations not observed in wild-type animals exposed to the same aggression. It was not analyzed, however, whether the cells forming microcysts in this model had both Pkd1 alleles mutated or not. At present, therefore, most investigators agree with the proposed model whereby IR may represent a third hit in mature kidneys with $P k d 1$ inactivation, reactivating kidney developmental programs and cell proliferation that can create the cellular environment required for rapid and diffuse cyst formation.

Abnormalities in planar cell polarity (PCP) have also been proposed to participate in cyst formation and growth, by perturbing the process of organized epithelial cell division along the longitudinal axis, necessary for tubule formation and elongation $(29,30)$. A recent report, however, concluded that the loss of oriented cell division is not sufficient to initiate cyst formation (31). This study with $P k d 1$ and $P k d 2$ mutated mouse models revealed that pre-cystic cells did not show lack of oriented division while they lost it after the initiation of tubular dilatation. Homozygous mice for a mutation in Pkhd1, the orthologue of the human PKHD1 gene (mutated in autosomal recessive polycystic kidney disease), in turn, lost oriented cell division, but did not progress to renal cyst formation. These observations indicate that disoriented cell division is not required to start cystogenesis and is insufficient to lead to renal cysts in the mentioned animal models. PCP defects, therefore, seem to contribute to cystic growth, but are not the primary event that triggers cystogenesis.

\section{Cellular and transport abnormalities}

The ADPKD cyst-lining epithelial cells are characterized by high rates of proliferation and apoptosis, dedifferentiation, planar polarity defects, and a secretory behavior (1). Extracellular matrix remodeling is also part of this phenotype. Several findings support essential roles for the polycystins in the control of cell proliferation and in the maintenance of a differentiated phenotype of renal tubular epithelium. In support to those findings, the reduction of PC1 or PC2 below a critical level is accompanied by an abnormal cellular phenotype, characterized by increased cell proliferation and apoptosis, disturbed planar cell polarity, extracellular matrix alterations, and a phenotypic change in salt and fluid transport from a reabsorptive to a secretory pattern. The PC1 and PC2 subcellular expression profiles, in turn, are consistent with these mediatory roles.

Unlike normal collecting duct principal cells, in cystic epithelium $\mathrm{Cl}^{-}$is secreted, being driven across the basolateral membrane by a $\mathrm{Na}^{+} \mathrm{K}^{+} 2 \mathrm{Cl}^{-}$cotransporter, a process that follows the gradient established by $\mathrm{Na}^{+} \mathrm{K}^{+} \mathrm{ATPase}$. Its exit across the apical membrane, in turn, occurs through the cAMP-triggered $\mathrm{Cl}^{-}$channel cystic fibrosis transmembrane conductance regulator (CFTR). Interestingly, the required $\mathrm{K}^{+}$recycling across the basolateral membrane may happen through the $\mathrm{K}^{+}$channel $\mathrm{KCa} 3.1$ (32). The progressive $\mathrm{Cl}^{-}$accumulation within the cyst lumen, therefore, drives $\mathrm{Na}^{+}$and $\mathrm{H}_{2} \mathrm{O}$ secretion following transepithelial potential and osmotic gradients.

The molecular basis for these phenotypic changes is largely unclear, but the recent identification of altered signaling pathways in the disease has provided some initial clues. An important observation is that PC1 complexes with E-cadherin and $\alpha-, \beta$ - and $\gamma$-catenins at cell adherens junctions. Depletion of $\mathrm{Ca}^{2+}$, however, retains PC1 and E-cadherin in the cytoplasm, while its repletion is followed by the colocalization of both proteins at cell-cell contacts (33). These data support a current model whereby PC1 may modulate the intensity of cell adhesion by regulating the formation of appropriate adherens junctions. In this context, it is key to note that in ADPKD the PC1/E-cadherin complex is disrupted.

PC1 and PC2 are also expressed in endothelium and vascular smooth muscle cells. These findings support the observed endothelial dysfunction and reduced nitric oxide generation in ADPKD patients, as well as an increased contractile response of the arterial vasculature in $P k d 2^{+/-}$mice (34). Amore recent study, in addition, showed that polycystins play a complex role in the maintenance of vascular integrity and that $P k d 1$ and $P k d 2$ are necessary for the normal development of the placenta in mice (35).

The fact that the polycystins participate in several cell signaling pathways makes the process of elucidating these molecular mechanisms a complex task. In spite of this scenario, some interventions anchored on alterations of recognized pathways have been successful in blocking or slowing the cystic kidney phenotype in animal models of polycystic kidney disease, orthologous and nonorthologous to human ADPKD. Such signaling pathways will be discussed in the following sections.

\section{Ciliary dysfunction and abnormal intracellular $\mathrm{Ca}^{2+} / \mathrm{CAMP}$ signaling}

PC1 and PC2 have been shown to colocalize in the primary cilium, an apical sensory organelle bulged to the lumen in renal tubular epithelial cells. The PC1-PC2 complex is thought to function as a mechano- or chemosensor in the 
cilia, translating extracellular stimuli into $\mathrm{Ca}^{2+}$ influx through $\mathrm{PC} 2$. This initial influx triggers significant $\mathrm{Ca}^{2+}$ release from intracellular stores, which modulates biological processes such as cell proliferation, differentiation, apoptosis, and gene expression. The ryanodine and the inositol 1,4,5-triphosphate receptors seem to participate in the PC2-related effects on the cytoplasmic $\mathrm{Ca}^{2+}$ level $(36,37)$. It is currently admitted that PC2 in the ER participates in intracellular $\mathrm{Ca}^{2+}$ homeostasis. A recent study suggests that interaction with syntaxin 5 inactivates $\mathrm{PC} 2$, preventing $\mathrm{Ca}^{2+}$ leakage from the ER (38). The $\mathrm{Ca}^{2+}$ release from intracellular stores is reduced in the settings of $\mathrm{PC} 2$ absence, haploinsufficiency or overexpression. Cyst cells, therefore, show defective $\mathrm{Ca}^{2+}$ signaling in response to fluid flow-dependent ciliary bending and diminished $\mathrm{ER} \mathrm{Ca}^{2+}$ stores, as well as decreased intracellular $\mathrm{Ca}^{2+}$ concentration in some circumstances. In this context, there is a defective homeostasis of intracellular $\mathrm{Ca}^{2+}$ in ADPKD, which apparently leads to intracellular accumulation of cAMP (Figure 3) (1). This reduced activity of the polycystin complex and consequent decrease in intracellular $\mathrm{Ca}^{2+}$ concentration, in fact, stimulates $\mathrm{Ca}^{2+}$-inhibitable adenylyl cyclase 6 and inhibits $\mathrm{Ca}^{2+} /$ calmodulin-dependent phosphodiesterase 1 , favoring the intracellular generation and accumulation of cAMP. Interestingly, the myocyte enhancer factor 2C (MEF2C) and histone deacetylase were found to be targets of the fluid stress sensing related to the PC1-PC2 complex in mouse kidney epithelial cells. Kidneyspecific Mefc2 knockout, in fact, determined significant tubule dilatation and renal cysts (39).

In contrast to normal kidney epithelial cells, in which cAMP inhibits proliferation, ADPKD cells present an aberrant proliferative response to cAMP. The intracellular accumulation of cAMP in PKD cells, in fact, is followed by the recruitment and phosphorylation of a series of proteins and activates the mitogen-activated protein kinase/ERK (MAPK/ERK) pathway (Figure 3), promoting cell proliferation and $\mathrm{Cl}^{-}$and fluid secretion, processes that act on cyst growth. In support to these findings, transfection of collecting duct principal cells with a dominant negative construct of the PC1 C-terminus led to cAMP-stimulated proliferation and activation of B-Raf and ERK, a process that was inhibited by a $\mathrm{Ca}^{2+}$ ionophore (40). The process of lowering intracellular $\mathrm{Ca}^{2+}$ in wild-type cells, in addition, triggers the abnormal proliferative response to cAMP (40). Procedures that promoted an increase in intracellular $\mathrm{Ca}^{2+}$ in human autosomal dominant and autosomal recessive polycystic kidney disease (ADPKD and ARPKD) cells, moreover, were effective in returning them to a normal proliferation phenotype (41).

\section{Additional signaling pathways altered in ADPKD}

Different lines of evidence demonstrate a direct link between polycystins and cell cycle regulation. A classical study reported that activation of PC1, following a PC2-dependent process, activates JAK2, leading to phosphorylation and formation of STAT1 homodimers. These dimers, in turn, translocate to the cell nucleus and bind to the $p 21$ gene promoter, up-regulating its product (42). The p21 up-regulation inhibits Cdk2 activity, determining cell cycle arrest in G0/G1. This mechanism may significantly contribute to the inhibitory effect of PC1 on cell proliferation. PC1 also contains a conserved trimeric G-protein activation domain, leading Gprotein subunits to mediate the PC1-related activation of the C-Jun N-terminal kinase (JNK) and AP-1 transcription factor (43). The alteration in AP-1 activity reported in ADPKD, in fact, may contribute to the abnormalities in cell differentiation, proliferation and apoptosis observed in this disease. Other investigators have reported that the activation of JNK by PC1 occurs via protein kinase C-alpha (PKC- $\alpha$ ). A recent study, in turn, revealed that the activator of G-protein signaling 3 may regulate $\mathrm{G} \alpha$ subunit function in a receptor-independent manner, modulating the function of epithelial cells in PKD.

PC1 has been reported to favor the stabilization of the tuberin-hamartin complex, inhibiting mammalian target for rapamycin (mTOR) activity. This process can be secondary to the PC1 interaction with the TSC2 gene product, tuberin, at the plasma membrane level, preventing its phosphorylation by protein kinase $B(A k t)$ and consequently allowing the tuberin-hamartin complex to inhibit mTOR. Alternatively, PC1 can reduce tuberin ERK-dependent phosphorylation (44), keeping the tuberin-hamartin complex stable. In ADPKD, the disruption of such mechanisms activates mTOR and promotes cell proliferation (Figure 3). PC2, in turn, can also decrease cell proliferation by interacting with pancreatic elF2 $\alpha$ kinase (PERK) at the ER level, promoting phosphorylation of the translation initiation factor 2 alpha (elF2 $\alpha)(45)$. The three proteins PC2, PERK and elF2 $\alpha$, in fact, were found to be in the same complex. Increased apoptosis is another key feature ofADPKD. It is currently admitted that resistance to apoptosis induced by PC1 is a process probably dependent on GiCPR activation of phosphatidylinositol 3-kinase and Akt.

As previously pointed out, the disruption of PCP appears to play a role in ADPKD cyst expansion $(29,30)$. The molecular basis of these abnormalities in cell division orientation has received some clues in the recent years. A series of observations have revealed activation of the canonical $\beta$-catenin-dependent Wnt signaling pathway as an early effect of $P k d 1$ gene disruption (46) and implicate both canonical and non-canonical Wnt signaling pathways in cyst formation. In agreement with such findings, a more recent study has reported increased levels of $\beta$-catenin in cultured $P k d 2-$ knockout mouse cells (Figure 3 ). It has not been clarified, however, whether this $\beta$-catenin increase is determined by the absence of PC2 itself or by PC2-dependent PC1 dysregulation. Curiously, it has been shown that secreted Frizzled-related protein 4 (sFRP4) is up-regulated in ADPKD and in Pkd2-/- mice (47) (Figure 3). The fact that it inhibits only part of the components of the canonical Wnt signaling pathway probably explains why $\beta$-catenin levels can increase in PKD.

Two recent studies have shown that $P k d 1$ and $P k d 2$ 


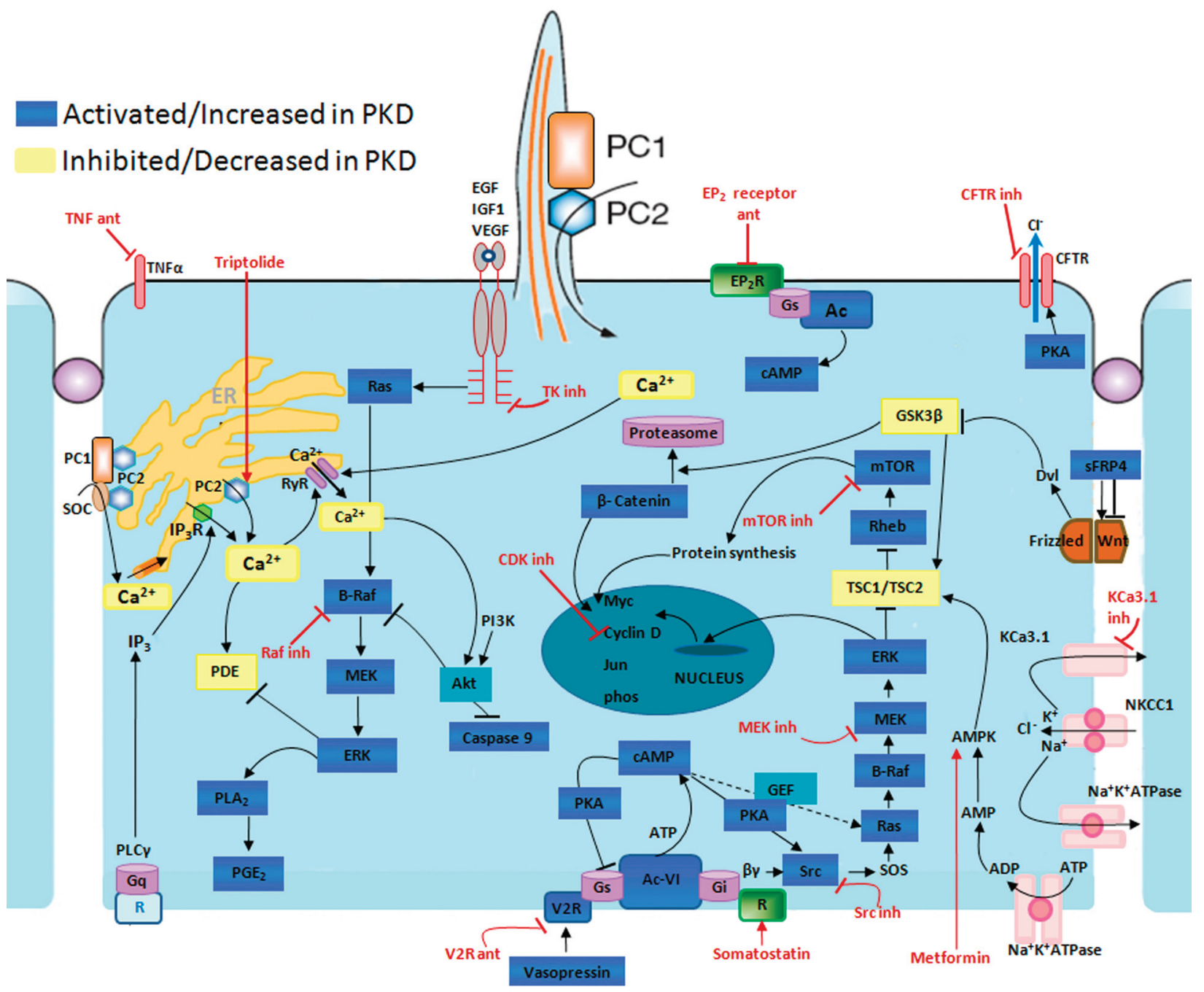

Figure 3. Disrupted pathways in polycystic kidney disease (PKD) and points of potential therapeutic intervention with specific agents. Agents associated with potential therapeutic interventions are represented in red; ant $=$ antagonist; inh $=$ inhibitor. $\mathrm{AC}-\mathrm{VI}=$ adenylate cyclase $6 ;$ Akt = protein kinase B; AMPK = AMP kinase; B-Raf = v-raf murine sarcoma viral oncogene homolog B1; CDK = cyclin-dependent kinase; CFTR = cystic fibrosis transmembrane conductance regulator; $E G F=$ epidermal growth factor; $E P_{2} R=E$-prostanoid receptor 2; ERK = extracellular signal-regulated protein kinase; GSK3 = glycogen synthase kinase 3; IGF1 = insulin-like growth factor $1 ; \mathrm{IP}_{3} \mathrm{R}=$ inositol 1,4,5-trisphosphate receptor; $\mathrm{MAPK}=$ mitogen-activated protein kinase; $\mathrm{mTOR}=$ mammalian target for rapamycin; $\mathrm{PC} 1$ = polycystin-1; PC2 = polycystin-2; $\mathrm{PDE}=$ phosphodiesterase; $\mathrm{PGE}_{2}=$ prostaglandin $\mathrm{E} 2 ; \mathrm{PI} \mathrm{K}=$ phosphatidylinositol 3-kinase; PKA = protein kinase $\mathrm{A} ; \mathrm{PLA}_{2}=$ phospholipase $\mathrm{A} 2 ; \mathrm{PLC} \gamma=$ phospholipase $\mathrm{C}-\mathrm{\gamma} 2 ; \mathrm{R}=$ somatostatin sst2 receptor; Rheb = ras homolog enriched in brain; RyR = ryanodine receptor; sFRP4 = secreted Frizzled-related protein 4; TK = tyrosine kinase; TNF- $\alpha=$ tumor necrosis factor- $\alpha$; TSC = tuberous sclerosis proteins tuberin (TSC2) and hamartin (TSC1); V2R = vasopressin V2 receptor; VEGF = vascular endothelial growth factor. (Adapted from a schema previously used by Vicente E. Torres and Peter C. Harris, with permission).

haploinsufficiency have a significant impact on the renal cell repair response to IR. Such studies revealed an increased susceptibility to ischemia/reperfusion insult in $P k d 1^{+/-}$and $P k d 2^{+/-}$mice compared with their corresponding wild-type controls $(28,48)$. Increased cell proliferation, apoptosis, inflammation, and late fibrosis were observed in the haploinsufficient animals, as well as a lower level of p21 expression following the IR insult. Progress has also been made in the understanding of dysregulation of metalloproteinase inhibitor and matrix-degrading enzyme expression. Cyst epithelial cells were found to generate large quantities of laminin, collagen I and III, transforming growth factor-beta (TGF- $\beta$ ) and periostin, which are deposited around the cystic structures. Laminin- 5 is markedly expressed in the extracellular matrix of ADPKD kidneys but not in control organs, while ADPKD cells produce and secrete this mol- 
ecule. Laminin-5, moreover, is a significant regulator of ADPKD cell proliferation and cyst formation. A recent study by Hassane et al. (49) has indicated that TGF- $\beta$ signaling does not play a role in the beginning of cystogenesis, but participates significantly during cyst expansion and fibrogenesis along the progression of ADPKD (49). An analysis performed in the PCK rat, a model orthologous to human ARPKD, suggests, in addition, that epithelial cells in renal cysts develop mesenchymal characteristics in response to cyst growth, a process that may contribute to progressive kidney fibrosis.

\section{Preclinical studies and therapeutic prospects based on molecular and cellular pathogenesis}

A number of preclinical studies based on the pathogenetic findings described here were carried out in the past decade, trying to prevent PKD development or to slow the progression of renal cystic disease. These analyses have been performed in animal models orthologous and nonorthologous to human renal cystic diseases. In this review, priority will be given to the results obtained in orthologous models. Notably, some of the data have been strong enough to justify large clinical trials in ADPKD patients, including already concluded, ongoing and planned studies.

Renal cysts originate predominantly from collecting ducts in ADPKD, at least in the early stages. In the principal cells of collecting ducts, the vasopressin/V2 receptor system is a major source of cAMP generation. Treatment with a $\mathrm{V} 2$ receptor antagonist inhibited cyst formation and the progression of the polycystic kidney phenotype in the PCK rat (50) and in the Pkd2WS25/- mouse (51), supporting a central role of cAMP in PKD (Figure 3). A phase-3 multicenter clinical trial with Tolvaptan, a vasopressin V2 receptor antagonist, is ongoing in ADPKD patients. Furthermore, the inhibition of vasopressin release by a very high water intake in the PCK rat showed a protective effect on the renal cystic disorder. In accordance to these findings, an antagonist of the endothelin B receptor increased the cAMP renal level and worsened the cystic phenotype in $P k d 2$ WS25/- mice through a mechanism that apparently includes an increased vasopressin action.

Several effects of somatostatin may hinder cyst development both in kidney and liver. These effects include inhibition of vasopressin-dependent cAMP generation at the collecting duct level via Gi-protein-coupled receptors, inhibition of secretin-dependent cAMP production and fluid secretion in cholangiocytes, and reduction of insulin-like growth factor 1 and vascular endothelial growth factor (VEGF) expression (Figure 3). The long-acting somatostatin analogue octreotide, in fact, improved the cystic renal and liver phenotypes of the PCK rat, emphasizing the significance of cAMP signaling in PKD (52). Positive results have been obtained with octreotide in ADPKD patients with polycystic kidneys and liver and in isolated autosomal dominant polycystic liver disease (ADPLD) patients (53), but larger and longer clinical trials are required. In support to the $\mathrm{Ca}^{2+}$ cAMP signaling role in PKD, triptolide, a drug shown to be able to release $\mathrm{Ca}^{2+}$ through a process that depends on PC2, ameliorated the cystic disease in kidney-specific Pkd1 conditional knockout mice, attenuated the cystic phenotype in $P k d 1^{-/-}$mice during embryonic life, and blocked proliferation of $P k d 1^{-/-}$cells (Figure 3 ). Treatment with a type 2 calcimimetic, however, did not affect renal cAMP levels or cystogenesis in Pkd2WS25/- mice and PCK rats, a finding that might be explained by a decrease in extracellular $\mathrm{Ca}^{2+}$ or by the lack of $\mathrm{Ca}^{2+}$-sensing receptors in the cortical and outer medullary portions of collecting ducts.

Focusing on inhibition of mTOR (Figure 3), recent studies showed that sirolimus reduced the cystic burden and preserved renal function in Pkd1cond/cond:NestinCre mice (54), and also reduced the progression of cystic disease in Pkd2WS25/- mice. The conclusion of a recent clinical trial using sirolimus in ADPKD patients with early renal disease, however, did not show blockade of kidney growth (55), while another large clinical trial with everolimus in ADPKD patients with more advanced disease reported diminishment in kidney volume increase but no reduction in the rate of renal function decline (56). Based on its stimulating effect upon AMP-activated protein kinase, metformin can inhibit CFTR and inhibit mTOR by phosphorylating tuberin (Figure $3)$. Notably, metformin reduced the cystic phenotype in two mouse models orthologous to ADPKD1: Pkd1 $1^{\text {flox } /-; K s p: C r e ~}$ and $P k d 1^{\text {flox/-; }}$ CX:CreER with Cre induction at P9 or P10 (57). Inhibition of mTOR can also hinder the generation of VEGF and suppress its cellular effects. Interestingly, the VEGF receptor (VEGFR) inhibitor SU-5416 blocked cyst growth in liver but not in kidney in Pkd2WS25/- mice, whereas the administration of anti-VEGFR2 antibodies led to the formation of renal cysts in early-life CD-1 mice. In a recent report, Qin et al. (58) showed that mTOR hyperactivation in $P k d 1^{-/-}$mouse cells occurs due to a lack of appropriate ubiquitination and degradation of the hepatocyte growth factor (HGF) receptor c-Met following HGF stimulation. Treatment of $P k d 1^{-/-}$kidneys in organ culture with a c-Met inhibitor indeed reduced the size and number of cysts.

A series of drugs that reduce cell proliferation have been studied in PKD animal models and have been shown to promote improvement of cystic phenotype. Such agents include inhibitors of Src kinase, epidermal growth factor receptor 1 (Erb-B1) and Erb-B2 tyrosine kinase and MEK (Figure 3). Sorafenid, a small molecule Raf inhibitor, inhibits cAMP-dependent proliferation and in vitro cyst growth of human ADPKD cystic cells. The cyclin-dependent kinase inhibitor roscovitine has also been effective by arresting the cell cycle, inhibiting apoptosis and regulating transcription. This drug has significantly reduced cyst formation and attenuated renal disfunction in the juvenile cystic kidneys (jck) and congenital polycystic kidney (cpk) mice, two PKD 
animal models non-orthologous to human ADPKD or ARPKD (59). Pioglitazone, a peroxisome proliferator-activated receptor Y (PPAR-Y) agonist, ameliorated the cardiac defects, decreased renal cystogenesis and increased survival in $\mathrm{Pkd1}^{-/-}$embryos, and inhibited kidney and liver cyst growth in the PCK rat. Genz-123346, a drug that inhibits the accumulation of glucosylceramide, has also shown positive results in an animal model orthologous to human ADPKD1. Recent studies, furthermore, have reported that the inhibitor of histone deacetylase valproic acid reduced cyst formation and the rate of renal function decline in a Pkd1 flox/flox;Pkhd1:Cre mouse model (60). Trichostatin A, another histone deacetylase inhibitor, was also capable of reducing cyst formation in Pkd2/- mouse embryos (39).

Turning to transepithelial $\mathrm{Cl}^{-}$and fluid secretion (Figure 3), CFTR and KCa3.1 inhibitors were tested. CFTR inhibition reduced the rate of cyst growth and preserved renal function in $P k d 1^{f l o x /-;}$ Ksp:Cre mice, while the KCa3.1 inhibitor TRAM-34 inhibited transepithelial $\mathrm{Cl}^{-}$secretion stimulated by forskolin in ADPKD cell polarized monolayers and cyst formation and growth by ADPKD cells cultured in collagen gel.

Tumor necrosis factor- $\alpha$ (TNF- $\alpha$ ) is found in the fluid of human ADPKD cysts and its mRNA level increases progressively with time in the kidneys of the cpk mouse cystic model. Intraperitoneal TNF- $\alpha$ injection, moreover, leads to cyst formation in $P k d 2^{+/-}$mice. This pro-cystic effect (Figure 3 ) is corroborated by the observation that etanercept, a TNF- $\alpha$ blocker, prevented cyst formation in this orthologous animal model. Additional data obtained in a human epithelial cell-culture system derived from normal and ADPKD kidneys, developed in a three-dimensional gel matrix, suggest that prostaglandin E2 (PGE2), present in the cyst fluid, binds to apical EP2 receptors and stimulates its expression, increasing cAMP production and promot-

\section{References}

1. Torres VE, Harris PC. Autosomal dominant polycystic kidney disease: the last 3 years. Kidney Int 2009; 76: 149-168.

2. Pirson Y. Extrarenal manifestations of autosomal dominant polycystic kidney disease. Adv Chronic Kidney Dis 2010; 17: 173-180.

3. Chapman AB, Guay-Woodford LM, Grantham JJ, Torres VE, Bae KT, Baumgarten DA, et al. Renal structure in early autosomal-dominant polycystic kidney disease (ADPKD): The Consortium for Radiologic Imaging Studies of Polycystic Kidney Disease (CRISP) cohort. Kidney Int 2003; 64: 10351045.

4. Pei Y, Paterson AD, Wang KR, He N, Hefferton D, Watnick T, et al. Bilineal disease and trans-heterozygotes in autosomal dominant polycystic kidney disease. Am J Hum Genet 2001; 68: 355-363

5. Hateboer N, Dijk MA, Bogdanova N, Coto E, Saggar-Malik AK, San Millan JL, et al. Comparison of phenotypes of polycystic kidney disease types 1 and 2. European PKD1-PKD2 ing cystogenesis (Figure 3). EP2 receptor antagonists, therefore, may become a potential therapeutic target for ADPKD. The cytochrome P450 metabolite of arachidonic acid 20-hydroxyeicosatetraenoic (20-HETE) is generated in larger amounts in microsomes of the bpk mouse kidney, a recessive model non-orthologous to human ARPKD. The observation that inhibition of its synthesis by HET-0016 markedly reduced kidney size and prolonged survival in this mouse model suggests that the endogenous metabolite 20-HETE mediates cell proliferation in the formation of renal cysts.

\section{Conclusion and Perspectives}

The scientific reality regarding ADPKD, therefore, has gone through dramatic changes within the past two decades, particularly in recent years. In this new scenario, the progressive elucidation of its molecular and cellular pathogenesis is substantially extending our understanding of biological systems and mechanisms, as well as opening promising therapeutic perspectives. The animal and cellular models have been playing central roles in this process, markedly contributing to the gradual understanding of PKD pathogenesis and allowing essential preclinical studies. Such knowledge accumulation, in turn, has already led to the performance of initial information-based clinical trials. The progressive genetic, molecular and cellular sophistication of biological models will keep defining the scientific progress in this field.

\section{Acknowledgments}

Research supported by FAPESP (\#2009/13926-3 to L.F. Onuchic and \#2010/10425-0 to A.P. Bastos).

Study Group. Lancet 1999; 353: 103-107.

6. Harris PC, Bae KT, Rossetti S, Torres VE, Grantham JJ, Chapman $A B$, et al. Cyst number but not the rate of cystic growth is associated with the mutated gene in autosomal dominant polycystic kidney disease. J Am Soc Nephrol 2006; 17: 3013-3019.

7. Hughes J, Ward CJ, Peral B, Aspinwall R, Clark K, San Millan JL, et al. The polycystic kidney disease 1 (PKD1) gene encodes a novel protein with multiple cell recognition domains. Nat Genet 1995; 10: 151-160.

8. Mochizuki T, Wu G, Hayashi T, Xenophontos SL, Veldhuisen $\mathrm{B}$, Saris JJ, et al. PKD2, a gene for polycystic kidney disease that encodes an integral membrane protein. Science 1996; 272: 1339-1342.

9. Rossetti S, Kubly VJ, Consugar MB, Hopp K, Roy S, Horsley $\mathrm{SW}$, et al. Incompletely penetrant PKD1 alleles suggest a role for gene dosage in cyst initiation in polycystic kidney disease. Kidney Int 2009; 75: 848-855. 
10. Feng S, Okenka GM, Bai CX, Streets AJ, Newby LJ, DeChant BT, et al. Identification and functional characterization of an N-terminal oligomerization domain for polycystin-2. J Biol Chem 2008; 283: 28471-28479.

11. Low SH, Vasanth $\mathrm{S}$, Larson $\mathrm{CH}$, Mukherjee S, Sharma $\mathrm{N}$, Kinter MT, et al. Polycystin-1, STAT6, and P100 function in a pathway that transduces ciliary mechanosensation and is activated in polycystic kidney disease. Dev Cell 2006; 10: 57-69.

12. Kottgen M, Benzing T, Simmen T, Tauber R, Buchholz B, Feliciangeli S, et al. Trafficking of TRPP2 by PACS proteins represents a novel mechanism of ion channel regulation. EMBO J 2005; 24: 705-716.

13. Hanaoka K, Qian F, Boletta A, Bhunia AK, Piontek K, Tsiokas $\mathrm{L}$, et al. Co-assembly of polycystin- 1 and -2 produces unique cation-permeable currents. Nature 2000; 408: 990-994.

14. Chapin HC, Rajendran V, Caplan MJ. Polycystin-1 surface localization is stimulated by polycystin-2 and cleavage at the $\mathrm{G}$ protein-coupled receptor proteolytic site. Mol Biol Cell 2010; 21: 4338-4348

15. Lu W, Shen X, Pavlova A, Lakkis M, Ward CJ, Pritchard L, et al. Comparison of Pkd1-targeted mutants reveals that loss of polycystin-1 causes cystogenesis and bone defects. Hum Mol Genet 2001; 10: 2385-2396.

16. Yu S, Hackmann K, Gao J, He X, Piontek K, Garcia-Gonzalez MA, et al. Essential role of cleavage of Polycystin-1 at G protein-coupled receptor proteolytic site for kidney tubular structure. Proc Natl Acad Sci U S A 2007; 104: 1868818693.

17. Lal M, Song X, Pluznick JL, Di Giovanni V, Merrick DM, Rosenblum ND, et al. Polycystin-1 C-terminal tail associates with beta-catenin and inhibits canonical Wnt signaling. Hum Mol Genet 2008; 17: 3105-3117.

18. Qian F, Watnick TJ, Onuchic LF, Germino GG. The molecular basis of focal cyst formation in human autosomal dominant polycystic kidney disease type I. Cell 1996; 87: 979-987.

19. Wu G, Markowitz GS, Li L, D'Agati VD, Factor SM, Geng L, et al. Cardiac defects and renal failure in mice with targeted mutations in Pkd2. Nat Genet 2000; 24: 75-78.

20. Lantinga-van Leeuwen I, Dauwerse JG, Baelde HJ, Leonhard WN, van de Wal A, Ward CJ, et al. Lowering of Pkd1 expression is sufficient to cause polycystic kidney disease. Hum Mol Genet 2004; 13: 3069-3077.

21. Nishio S, Hatano M, Nagata M, Horie S, Koike T, Tokuhisa $\mathrm{T}$, et al. Pkd1 regulates immortalized proliferation of renal tubular epithelial cells through p53 induction and JNK activation. J Clin Invest 2005; 115: 910-918.

22. Thivierge C, Kurbegovic A, Couillard M, Guillaume R, Cote O, Trudel M. Overexpression of PKD1 causes polycystic kidney disease. Mol Cell Biol 2006; 26: 1538-1548.

23. Park EY, Sung YH, Yang MH, Noh JY, Park SY, Lee TY, et al. Cyst formation in kidney via B-Raf signaling in the PKD2 transgenic mice. J Biol Chem 2009; 284: 7214-7222.

24. Piontek K, Menezes LF, Garcia-Gonzalez MA, Huso DL, Germino GG. A critical developmental switch defines the kinetics of kidney cyst formation after loss of Pkd1. Nat Med 2007; 13: 1490-1495

25. Patel V, Li L, Cobo-Stark P, Shao X, Somlo S, Lin F, et al. Acute kidney injury and aberrant planar cell polarity induce cyst formation in mice lacking renal cilia. Hum Mol Genet 2008; 17: 1578-1590

26. Takakura A, Contrino L, Beck AW, Zhou J. Pkd1 inactivation induced in adulthood produces focal cystic disease. J Am Soc Nephrol 2008; 19: 2351-2363.

27. Takakura A, Contrino L, Zhou X, Bonventre JV, Sun Y, Humphreys $\mathrm{BD}$, et al. Renal injury is a third hit promoting rapid development of adult polycystic kidney disease. Hum Mol Genet 2009; 18: 2523-2531.

28. Bastos AP, Piontek K, Silva AM, Martini D, Menezes LF, Fonseca JM, et al. Pkd1 haploinsufficiency increases renal damage and induces microcyst formation following ischemia/reperfusion. J Am Soc Nephrol 2009; 20: 2389-2402.

29. Fischer E, Legue E, Doyen A, Nato F, Nicolas JF, Torres V, et al. Defective planar cell polarity in polycystic kidney disease. Nat Genet 2006; 38: 21-23.

30. Luyten A, Su X, Gondela S, Chen Y, Rompani S, Takakura A, et al. Aberrant regulation of planar cell polarity in polycystic kidney disease. J Am Soc Nephrol 2010; 21: 1521-1532.

31. Nishio S, Tian X, Gallagher AR, Yu Z, Patel V, Igarashi P, et al. Loss of oriented cell division does not initiate cyst formation. J Am Soc Nephrol 2010; 21: 295-302.

32. Albaqumi M, Srivastava S, Li Z, Zhdnova O, Wulff H, Itani $\mathrm{O}$, et al. $\mathrm{KCa} 3.1$ potassium channels are critical for CAMPdependent chloride secretion and cyst growth in autosomaldominant polycystic kidney disease. Kidney Int 2008; 74 : 740-749.

33. Markoff A, Bogdanova N, Knop M, Ruffer C, Kenis H, Lux P, et al. Annexin A5 interacts with polycystin-1 and interferes with the polycystin-1 stimulated recruitment of E-cadherin into adherens junctions. J Mol Biol 2007; 369: 954-966.

34. Qian Q, Hunter LW, Du H, Ren Q, Han Y, Sieck GC. Pkd2+/vascular smooth muscles develop exaggerated vasocontraction in response to phenylephrine stimulation. J Am Soc Nephrol 2007; 18: 485-493.

35. Garcia-Gonzalez MA, Outeda P, Zhou Q, Zhou F, Menezes LF, Qian F, et al. Pkd1 and Pkd2 are required for normal placental development. PLoS One 2010; 5: e12821.

36. Nauli SM, Alenghat FJ, Luo Y, Williams E, Vassilev P, Li X, et al. Polycystins 1 and 2 mediate mechanosensation in the primary cilium of kidney cells. Nat Genet 2003; 33: 129137.

37. Li Y, Wright JM, Qian F, Germino GG, Guggino WB. Polycystin 2 interacts with type I inositol 1,4,5-trisphosphate receptor to modulate intracellular $\mathrm{Ca}^{2+}$ signaling. J Biol Chem 2005; 280: 41298-41306.

38. Geng L, Boehmerle W, Maeda Y, Okuhara DY, Tian X, Yu $Z$, et al. Syntaxin 5 regulates the endoplasmic reticulum channel-release properties of polycystin-2. Proc Natl Acad Sci U S A 2008; 105: 15920-15925.

39. Xia S, Li X, Johnson T, Seidel C, Wallace DP, Li R. Polycystin-dependent fluid flow sensing targets histone deacetylase 5 to prevent the development of renal cysts. Development 2010; 137: 1075-1084.

40. Yamaguchi T, Wallace DP, Magenheimer BS, Hempson SJ, Grantham JJ, Calvet JP. Calcium restriction allows CAMP activation of the B-Raf/ERK pathway, switching cells to a cAMP-dependent growth-stimulated phenotype. J Biol Chem 2004; 279: 40419-40430.

41. Yamaguchi T, Hempson SJ, Reif GA, Hedge AM, Wallace DP. Calcium restores a normal proliferation phenotype in human polycystic kidney disease epithelial cells. J Am Soc Nephrol 2006; 17: 178-187.

42. Bhunia AK, Piontek K, Boletta A, Liu L, Qian F, Xu PN, et al. PKD1 induces p21(waf1) and regulation of the cell cycle 
via direct activation of the JAK-STAT signaling pathway in a process requiring PKD2. Cell 2002; 109: 157-168.

43. Parnell SC, Magenheimer BS, Maser RL, Zien CA, Frischauf AM, Calvet JP. Polycystin-1 activation of C-Jun N-terminal kinase and $A P-1$ is mediated by heterotrimeric $G$ proteins. $J$ Biol Chem 2002; 277: 19566-19572.

44. Distefano G, Boca M, Rowe I, Wodarczyk C, Ma L, Piontek $\mathrm{KB}$, et al. Polycystin-1 regulates extracellular signal-regulated kinase-dependent phosphorylation of tuberin to control cell size through mTOR and its downstream effectors S6K and 4EBP1. Mol Cell Biol 2009; 29: 2359-2371.

45. Liang G, Yang J, Wang Z, Li Q, Tang Y, Chen XZ. Polycystin-2 down-regulates cell proliferation via promoting PERKdependent phosphorylation of elF2alpha. Hum Mol Genet 2008; 17: 3254-3262.

46. Happe $\mathrm{H}$, Leonhard WN, van der Wal A, van de Water B, Lantinga-van Leeuwen I, Breuning $\mathrm{MH}$, et al. Toxic tubular injury in kidneys from Pkd1-deletion mice accelerates cystogenesis accompanied by dysregulated planar cell polarity and canonical Wnt signaling pathways. Hum Mol Genet 2009; 18: 2532-2542.

47. Romaker D, Puetz M, Teschner S, Donauer J, Geyer M, Gerke $P$, et al. Increased expression of secreted frizzledrelated protein 4 in polycystic kidneys. J Am Soc Nephrol 2009; 20: 48-56.

48. Prasad S, McDaid JP, Tam FW, Haylor JL, Ong AC. Pkd2 dosage influences cellular repair responses following ischemia-reperfusion injury. Am J Pathol 2009; 175: 14931503.

49. Hassane S, Leonhard WN, van der Wal A, Hawinkels LJ, Lantinga-van Leeuwen I, ten Dijke P, et al. Elevated TGFbeta-Smad signalling in experimental Pkd1 models and human patients with polycystic kidney disease. J Pathol 2010; 222: 21-31.

50. Gattone VH, Wang X, Harris PC, Torres VE. Inhibition of renal cystic disease development and progression by a vasopressin V2 receptor antagonist. Nat Med 2003; 9: 1323-1326.

51. Torres VE, Wang X, Qian Q, Somlo S, Harris PC, Gattone $\mathrm{VH}$. Effective treatment of an orthologous model of auto- somal dominant polycystic kidney disease. Nat Med 2004; 10: 363-364.

52. Masyuk TV, Masyuk Al, Torres VE, Harris PC, Larusso NF. Octreotide inhibits hepatic cystogenesis in a rodent model of polycystic liver disease by reducing cholangiocyte adenosine 3',5'-cyclic monophosphate. Gastroenterology 2007; 132: 1104-1116.

53. Hogan MC, Masyuk TV, Page LJ, Kubly VJ, Bergstralh EJ, Li $\mathrm{X}$, et al. Randomized clinical trial of long-acting somatostatin for autosomal dominant polycystic kidney and liver disease. J Am Soc Nephrol 2010; 21: 1052-1061.

54. Shillingford JM, Piontek KB, Germino GG, Weimbs T. Rapamycin ameliorates PKD resulting from conditional inactivation of Pkd1. J Am Soc Nephrol 2010; 21: 489-497.

55. Serra AL, Poster D, Kistler AD, Krauer F, Raina S, Young J, et al. Sirolimus and kidney growth in autosomal dominant polycystic kidney disease. N Engl J Med 2010; 363: 820829.

56. Walz G, Budde K, Mannaa M, Nurnberger J, Wanner C, Sommerer $\mathrm{C}$, et al. Everolimus in patients with autosomal dominant polycystic kidney disease. N Engl J Med 2010; 363: 830-840.

57. Takiar V, Nishio S, Seo-Mayer P, King JD Jr, Li H, Zhang L, et al. Activating AMP-activated protein kinase (AMPK) slows renal cystogenesis. Proc Natl Acad Sci U S A 2011; 108: 2462-2467.

58. Qin S, Taglienti M, Nauli SM, Contrino L, Takakura A, Zhou $\mathrm{J}$, et al. Failure to ubiquitinate c-Met leads to hyperactivation of mTOR signaling in a mouse model of autosomal dominant polycystic kidney disease. J Clin Invest 2010; 120: 36173628.

59. Bukanov NO, Smith LA, Klinger KW, Ledbetter SR, Ibraghimov-Beskrovnaya O. Long-lasting arrest of murine polycystic kidney disease with CDK inhibitor roscovitine. Nature 2006; 444: 949-952.

60. Cao Y, Semanchik N, Lee SH, Somlo S, Barbano PE, Coifman $R$, et al. Chemical modifier screen identifies HDAC inhibitors as suppressors of PKD models. Proc Natl Acad Sci U S A 2009; 106: 21819-21824. 